\title{
Numerical simulation of coaxial turbulent jet with and without discrete particles
}

\author{
K. Kumaran \& R. Sadr \\ Micro Scale Thermo Fluids (MSTF) Laboratory, \\ Mechanical Engineering Program, \\ Texas A\&M University at Qatar, Qatar
}

\begin{abstract}
Three dimensional, steady, turbulent flow RANS simulations are carried out to study the near flow field characteristics of a particle laden coaxial water jet. Spherical glass particles of 240 micron diameter are added to the central jet flow at two mass loading ratios of $0.074 \%$ and $0.22 \%$. Numerical results for single phase flow are first compared with experimental data obtained using Molecular Tagging Velocimetry (MTV). The predictions of the axial velocity, axial turbulent intensity, correlation coefficient and vorticity are compared for singlephase and two-phase flow cases to bring out the effect of addition of particles on the fluid phase, while the numerical results show that the influence of the particles on the continuous phase flow field is minimal.

Keywords: coaxial turbulent jets, particle laden, numerical simulation.
\end{abstract}

\section{Introduction}

The study of particle laden, coaxial turbulent jets in a confined environment has been a subject of interest due to its implications in several applications such as jet mixers, sprays, particle separators, and combustion chambers in the field of chemical, industrial and mechanical engineering. The near field region of a coaxial jet, typically extending up five to six diameters from the nozzle exit, plays a crucial role in shaping the turbulent characteristics of the jet. This has a direct effect on the mixing process of the particle in a particle-laden coaxial jet. It is therefore important to understand the flow field in this region to better understand the details of particle-laden coaxial jets. A review of the literature pertaining to coaxial jet flows with and without the dispersed phase is presented next. 
Ko and Kwan [1] experimentally investigated the initial region of single phase coaxial jets at different mean velocity ratios. Based on the length of the inner and outer potential cores, they categorized the initial region of coaxial jet flow field into three zones namely, the inner merging zone, intermediate zone and the fully merged zone. Also, they reported that the outer mixing region resemble close to that in a single phase jet. Dahm et al. [2] investigated the vortex dynamics in the near field of the coaxial water jet for velocity ratios ranging from 0.59 to 4.16 . They reported that the sudden momentum change across the shear layer is an important aspect of the dynamics of coaxial jet. Later on, several studies $[3,4]$ were carried out to investigate the effect of inner wall thickness and diameter ratio on the flow field dynamics and mixing characteristics of single phase turbulent coaxial jets.

Mostafa et al. [5] investigated the initial region of coaxial jet flows with and without the glass beads of $110 \mu \mathrm{m}$ diameter and reported that the discrete particles attenuate the continuous phase turbulence. Fan et al. [6] investigated the effect of velocity ratio and particle mass loading on coaxial jets. The spreading rate of two phase coaxial jet was observed to be smaller than that of the single phase coaxial jet. Later, Fan et al. [7] confirmed these results using RANS simulations with $k-\varepsilon$ turbulence model. Recently, Virdung and Rasmuson [8] investigated the hydrodynamics of a liquid single jet dispersed with glass beads of $1.5 \mathrm{~mm}$ diameter. They carried out the numerical simulations with three different turbulent models and compared them with experimental data. It was reported that the realizable $k$ - $\varepsilon$ turbulence model was able to predict the mean axial velocity better with the drag correction term suggested by Brucato et al. [9] when compared to the other turbulent models. Despite the drag correction, the results were reported to be under- and over-predicted in the initial jet region. Stakic et al. [10] numerically investigated the influence of sand particles (of diameter $0.25 \mathrm{~mm}$ and $0.9 \mathrm{~mm}$ ) on the gas phase using the $k$ - $\varepsilon$ turbulence model. The particle size was reported to have an influence on the mechanism of momentum exchange between the continuous phase and the particles. However, the under prediction of the results was attributed to the assumption of isotropic nature of turbulence in the modeling. Later, in the same configuration, Sijercic et al. [11] numerically investigated the effect of turbulence anisotropy using a higher-order, Reynolds-stress turbulence model. However, the axial and radial turbulent intensities were under-predicted near the jet exit.

In recent years, the high end numerical methods such as large eddy simulation (LES) and direct numerical simulation (DNS) are used to solve the turbulent flow field either completely (DNS) or partially (LES) in contrast to RANS simulations wherein the turbulent eddies are modeled. The flow field predictions of particle laden coaxial turbulent jets using the LES (Liu et al. [12]) and DNS (Ahmed and Elghobashi [13]) methods have demonstrated to be superior to the RANS calculations. Nevertheless, these numerical methods are computationally too expensive to apply in practical applications at a time that RANS models are able to predict the two phase flows reasonably well within the scope of turbulent closure modeling. 
Although, several investigations were carried out in the past, most of them are case specific and demand further investigation to understand the performance of the modeling scheme to study the flow field. Thus in the present work, RANS calculations in conjunction with realizable $k$ - $\varepsilon$ turbulence model are carried out to investigate the effect of discrete particles on the continuous phase coaxial turbulent jet flow field at two different velocity ratios. The single phase numerical results are validated with experimental data of Sadr and Klewicki [14] herein after called the experiment. Later, predictions of the discrete phase results are compared with single phase results to highlight the influence of addition of particles on the continuous phase flow field.

\section{Experimental methodology}

Figure 1 shows a schematic of the experiment facility with its major system components. The test section measures $0.5 \mathrm{~m}$ in height and width and $0.6 \mathrm{~m}$ in length. The coaxial jet orifice located in the center of the upstream wall of the test section issues fluid horizontally into the test section. The central and annular jets are $d_{i}=30 \mathrm{~mm}$ and $d_{o}=75 \mathrm{~mm}$ in diameter, respectively. The wall thickness of the central jet nozzle, $t$, is $1.5 \mathrm{~mm}$. The central and the annular jet flows are driven by two pumps. The particles are made of clear spherical glass beads with a specific gravity of 2.46 and a mean diameter of $240 \mu \mathrm{m}$. A novel particle injection system, Sadr and Klewicki [15], is used to introduce particles into the central jet flow upstream of the jet orifice.

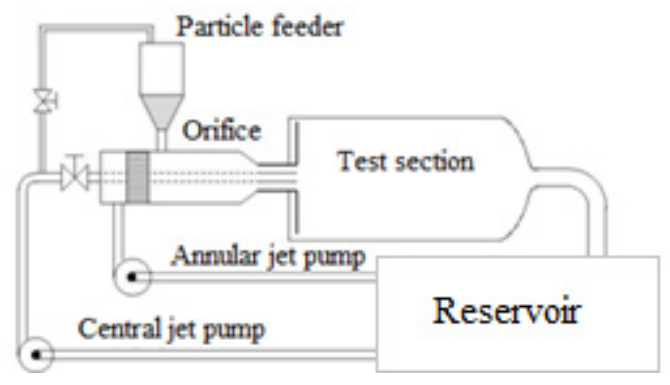

Figure 1: Schematic of the coaxial jet configuration.

Molecular tagging velocimetry (MTV), a whole field optical technique, is used to non-intrusively map the fluid flow velocities simultaneously at many points over a plane. It works by premixing the flowing medium and/or solid phase with molecules having long-lived luminescence lifetime. Typically, a pulsed UV laser is used to tag a region of interest in the flow. The tagged region is imaged at two successive times to record its displacement caused by the fluid flow. From this deformation, the kinematics of the flow may be obtained. The method is especially attractive for particulate flow studies since it does not require seeding, which may raise some complications with the presence of the 
solid phase. More details of the method and various parameters involved in it can be found in several review articles by Koochesfahani and Nocera [16].

Two different laser patterns are commonly used in MTV method: multiple lines and a grid. The major advantage of the multiline method is its fine spatial resolution. This method, hence, relies on finding the best fit to the laser line intensity profile near its peak. Figure 2 shows an example of the MTV images used by Sadr and Klewicki [17] to study the flow field in the particle- laden coaxial jet. The multiple line method is most accurate in flows dominated by a single velocity component. In this method, a series of coplanar MTV laser lines are used to measure single component of instantaneous velocity profiles.

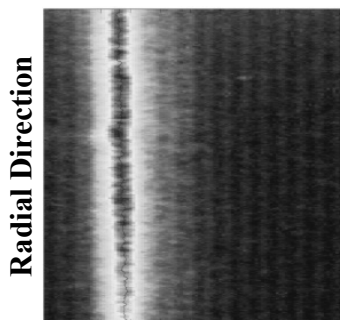

Axial direction

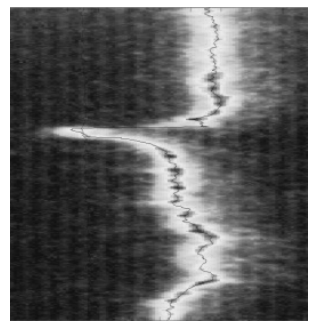

Axial direction

Figure 2: $\quad$ Sample undeformed (left) and deformed (right) MTV images at $\mathrm{x} / \mathrm{d}_{\mathrm{i}}=0.07$ and velocity ratio of 1.11 . Black line indicates the center of the laser line. Flow is from left to right.

\section{Numerical formulation and methodology}

Figure 3 shows a slice of the schematic of the computational domain considered here and it is only part of that shown in fig. 1. The central and coaxial jets issue into the test section at $\mathrm{x} / \mathrm{d}_{\mathrm{i}}=0$ where, $\mathrm{x}$ is the axial distance and $\mathrm{d}_{\mathrm{i}}$ is the inner pipe diameter. More details about the experimental facility are discussed in section 2. In this study, three dimensional, steady, incompressible, turbulent flow calculations are carried out in half domain (as shown in fig. 3) from symmetry considerations at two velocity ratios (ratio of outer to inner jet mean velocity) $\eta$ $=1.11$ and 0.18 . The inner jet velocity is held constant and the velocity ratio is varied by varying the outer jet velocity. In all the simulations, the working fluid is taken as water and mass and momentum equations (Reynolds-averaged Navier-Strokes (RANS)) are solved. The Reynolds stress, $\left(-\rho \overline{u_{i}^{\prime} u_{j}^{\prime}}\right)$ is modeled using the Boussinesq's approximation.

Initially, the single phase simulations were carried out using two turbulence models namely SST k- $\omega$ (Menter [18]) and realizable $k$ - $\varepsilon$ (Shih et al. [19]) turbulence models. Since the axial velocity profile and the turbulent intensity predictions at $\mathrm{x} / \mathrm{d}_{\mathrm{i}}=0$ are better with the realizable $k$ - $\varepsilon$ turbulence model, the two phase simulations are carried out with the realizable $k-\varepsilon$ turbulence model alone. Hence, the results obtained using realizable $k$ - $\varepsilon$ turbulence model alone is discussed here. The turbulent kinetic energy, $k$ and the dissipation rate, $\varepsilon$ are 
calculated using the realizable $k$ - $\varepsilon$ turbulence model with default model constants $\left(C_{1 \varepsilon}=1.44, C_{2}=1.9, \sigma_{k}=1.0\right.$ and $\left.\sigma_{\varepsilon}=1.2\right)$ where, $\sigma_{k}$ and $\sigma_{\varepsilon}$ are the turbulent prandtl numbers for the turbulent kinetic energy and dissipation rate respectively.

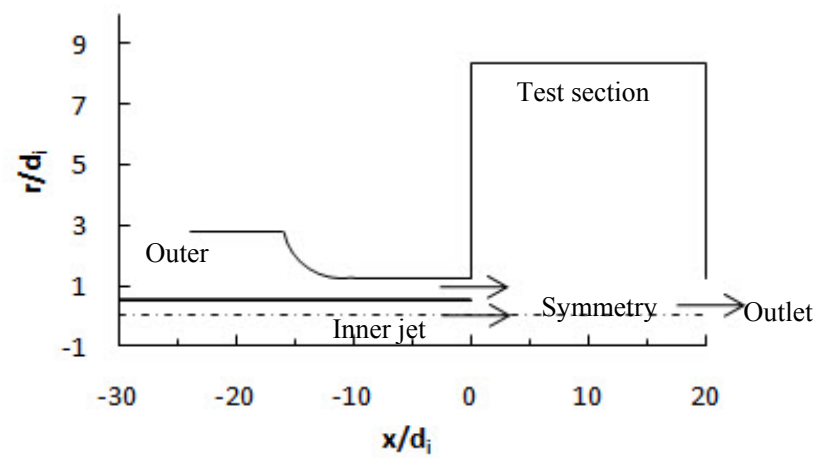

Figure 3: $\quad$ Schematic of the coaxial jet configuration (slice of a 3D domain).

The calculations with the discrete phase are carried out using EulerLagrangian approach where the continuous phase is solved in Eulerian mode and the discrete phase in Lagrangian mode. This is based on the fundamental assumption that the second phase (here discrete solid particles) volume fraction is small (typically $<5 \%$ ) when compared to the continuous phase in spite of the second phase having higher mass. The trajectory of the discrete phase is calculated based on the force balance of the particle as follows,

Particle Inertial force $=$ Drag force + gravity force + additional forces

The additional force could be due to virtual mass (when fluid density is more than the particle density), thermophoretic, brownian and Saffman's lift force. Based on the experimental condition, in this work only the drag force is accounted in the calculation. Consequently, the force balance results in the form,

$$
\frac{d u_{p}}{d t}=F_{D}\left(u-u_{p}\right), \text { where } F_{D}=\frac{18 \mu}{\rho_{p} d_{p}^{2}} \frac{C_{D} R e}{24} .
$$

Here, $u$ is the fluid phase velocity, $u_{p}$ is the particle velocity, $\mu$ is the molecular viscosity of the fluid, $\rho$ and $\rho_{p}$ are the fluid and particle densities respectively. $C_{D}$ is the Drag coefficient and Re is the relative Reynolds number, $\operatorname{Re} \equiv\left(\rho d_{p}\left|u_{p}-u\right|\right) /$ $\mu$. The drag force is calculated using the spherical drag law.

In discrete phase simulations, the effect of continuous phase turbulent velocity fluctuations on the particles is predicted using the stochastic tracking model. In addition to turbulent dispersion, two-way coupling is also considered to include the effect of particle fluctuations on the continuous phase. The particle-particle collision is not considered as the discrete phase concentration is below $0.3 \%$ (Hardalupas et al. [20]). The coupling between the continuous phase and the discrete phase is carried out using the particle-source-in-cell (PSI-CELL) 
approach. In all the simulations reported here, second order, upwind scheme is used for the spatial discretization and Roe-flux difference splitting scheme (Roe [21]) is used for the convective and diffusive flux terms. The pressure-velocity coupling is carried out using the coupled solver. All the calculations are carried out using the ANSYS-FLUENT [22].

\subsection{Boundary conditions}

The central and coaxial pipe inlets upstream of the test section inlet are defined as mass flow inlets where, the mass flow rates are adjusted until velocities at $\mathrm{x} / \mathrm{d}_{\mathrm{i}}$ $=0.07$ and $\mathrm{r} / \mathrm{d}_{\mathrm{i}}=0$ matches with the experimental values. Since the exact values of the inlet turbulent quantities at either of these pipe inlets are not available from experiments, a uniform specification of the turbulent intensity and the hydraulic diameter are specified. The Reynolds number for the inner jet is maintained at $4.1 \times 10^{4}$ (center line jet exit velocity $U_{c}=1.39 \mathrm{~m} / \mathrm{s}$ ) same as that of experiments. The outlet is defined as an outflow boundary condition with zero diffusive flux for all the variables and extrapolated from the interior of the domain. The mid-plane is specified as symmetry and all the walls are defined as stationary with no slip condition. At the plane of symmetry, the radial velocity and the radial gradients of other variables are defined to be zero.

For the discrete phase simulations, the discrete particles (glass beads of $240 \mu \mathrm{m}$ diameter) are injected in the central jet at two mass loading ratios (ratio of particle mass to continuous phase mass) of $0.074 \%$ and $0.22 \%$ similar to the experimental case of Sadr and Klewicki [17]. Volume fraction at this range is encountered in many practical applications. For example the primary stream of a pulverized coal burner operates at a volume fraction of $0.05 \%$ (Hardalupas et al. [20]) at standard condition.

\subsection{Convergence metrics}

All the calculations are carried out until the mass and momentum balances are within the acceptable limits. For all the results reported here, the difference in mass and momentum balance between the inlets and the outlet is within $1 \%$ and $5 \%$ respectively. All the results reported here is second order accurate.

\subsection{Grid independence}

The computational domain is completely meshed using the structured mesh. The near wall regions are resolved using the boundary layer mesh. In the test section, the first node from the wall is placed at about 50 microns and the following nodes are placed at different lengths using a progressive ratio. A top view of the node distribution on the test section symmetry plane and a close up view of the inner wall region are shown in fig. 4. A coarse mesh is generated first and then refined progressively until no significant change (i.e., less than 5\%) in the results was observed and that resulted in a total cell count of 799500, that herein after is called the base mesh. 


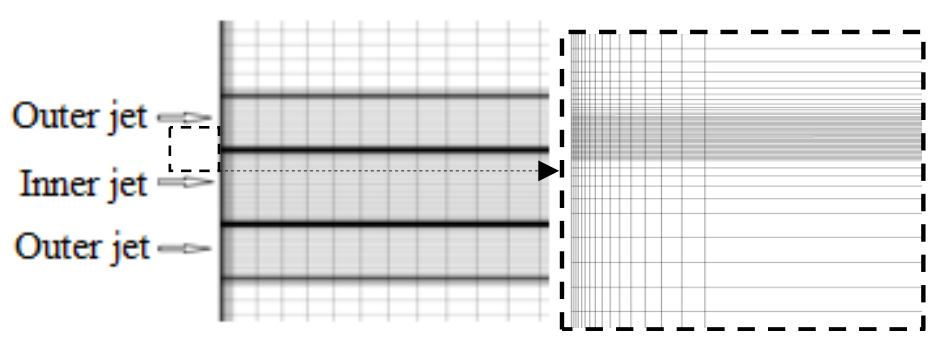

Figure 4: Top view of the node distribution in the symmetry plane (left) and a close up view of the inner wall region (right).

In order to attest to the fact that the results are grid independent, the base mesh was further refined in the region of interest, i.e., $x / \mathrm{d}_{\mathrm{i}}>0$, by reducing the distance between the cells in the radial and axial direction by half. This has resulted in a cell count of 1909500 . Since the wall refinement has been done adequately for the base mesh, the near wall refinement is retained the same in the refined mesh. As a result, the wall $\mathrm{y}^{+}$values between the meshes are almost the same $\left(\mathrm{y}^{+} \approx 40\right)$. Grid independence study is carried out only for the high velocity ratio. Figure 5 shows the comparison of radial profiles of the mean axial velocity and axial turbulent intensity at several locations downstream of the test section inlet between the base mesh and refined mesh.

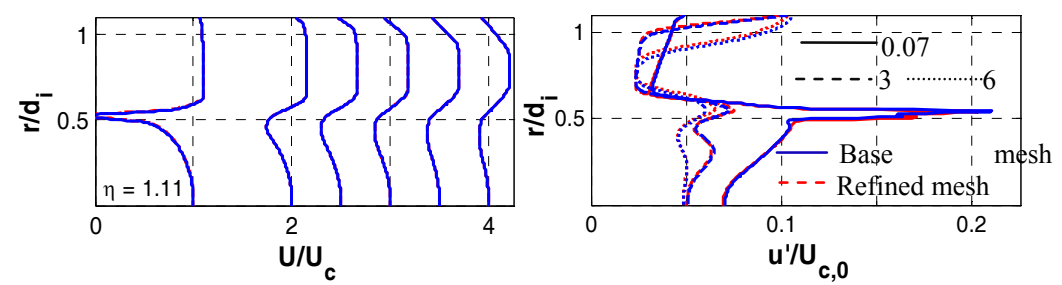

Figure 5: Comparison of axial mean velocity (left) and axial turbulent intensity (right) at different axial locations $\left(\mathrm{x} / \mathrm{d}_{\mathrm{i}}=0.07,2,3,4,5\right.$ and 6) between the base mesh (blue line) and refined mesh (red line) at velocity ratio, $\eta=1.11$. Here, $\mathrm{U}_{\mathrm{c}, 0}$ is the inner jet velocity at $\mathrm{x} / \mathrm{d}_{\mathrm{i}}=0$.

The variation of mean axial velocity between the meshes is almost the same. On the other hand, the difference in the variation of turbulent intensity profiles between the meshes is only marginal. These results clearly suggest that the base mesh is able to resolve the flow features adequately. Hence, all the results reported here are calculated using the base mesh. 


\section{Results and discussions}

In this section, results from the continuous phase simulations with and without considering the discrete particles at two velocity ratios $(\eta=1.11$ and 0.18$)$ are presented and discussed. First, the results of single-phase are compared with that of experimental data to establish the validity of the numerical procedure. Later, results from two phase flow calculations are compared with single phase results to highlight the influence of discrete particles on the continuous phase.

\subsection{Single phase flow}

Figure 6 shows the comparison of predicted radial profiles of the axial velocity at several axial locations downstream of the jet exits with experimental data. Here, the velocity profiles (except at $\mathrm{x} / \mathrm{d}_{\mathrm{i}}=0.07$ ) are offset by a factor of $0.5^{*} \mathrm{x} / \mathrm{d}_{\mathrm{i}}$ to show the variation. In both the cases, the inner jet $\left(0 \leq \mathrm{r} / \mathrm{d}_{\mathrm{i}} \leq 0.5\right)$ velocity profile at $\mathrm{x} / \mathrm{d}_{\mathrm{i}}=0.07$ is a fully developed velocity profile and the outer jet $\left(0.55 \leq \mathrm{r} / \mathrm{d}_{\mathrm{i}} \leq 1.25\right)$ is almost like a top-hat profile similar to that observed in the experiment. Since the velocity ratio is varied by varying the outer jet, the inner jet velocity profile is the same at all velocity ratios. The inlet jet velocity profile shows small deviation from experimental data in the region, $0.15<\mathrm{r} / \mathrm{d}_{\mathrm{i}}<0.4$. This could be due to the difference in the jet inlet conditions between the experiment and numerical simulation as mentioned in section 3.1. However, the velocity gradient near the inner jet wall region is predicted well (in the region $0.5 \leq \mathrm{r} / \mathrm{d}_{\mathrm{i}} \leq 0.55$ ).

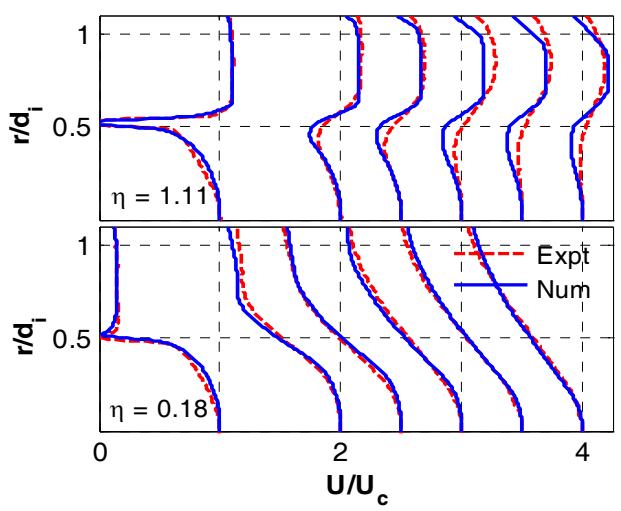

Figure 6: Radial variation of axial velocity at different axial locations (from left, $\mathrm{x} / \mathrm{d}_{\mathrm{i}}=0.07,2,3,4,5$ and 6 ) and velocity ratios.

In both velocity ratios, the outer jet velocity profile predictions at $\mathrm{x} / \mathrm{d}_{\mathrm{i}}=0.07$ are very close to experimental data. Further downstream, at $\eta=1.11$, the predictions show a greater deviation from experimental data in the inner shear region where the difference is close to $5 \%$ elsewhere, the deviation is within $3 \%$. This could be due to the over prediction of the wake effect when compared 
to experimental data. This difference decreases with decrease in velocity ratio. At low velocity ratio, the radial profile predictions agree with experimental data within $2 \%$.

Figure 7 shows the decay of the axial velocity and axial turbulent intensity comparison between the prediction and experimental data, along the centerline $\mathrm{r} / \mathrm{d}_{\mathrm{i}}=0$ (left) of the inner jet and along the midpoint of the coaxial jet $\mathrm{r} / \mathrm{d}_{\mathrm{i}}=0.86$ (right), both normalized by the inner jet exit velocity at $\mathrm{x} / \mathrm{d}_{\mathrm{i}}=0$. It is observed that in all the cases, the axial decay of mean axial velocity is predicted well within $5 \%$ of the experimental data. Along the centerline of the inner jet, the turbulent levels are under-predicted however, the trends are predicted well while at $\mathrm{r} / \mathrm{d}_{\mathrm{i}}=0.86$, the turbulent intensity levels are predicted closer to experimental data at both velocity ratios (near $\mathrm{x} / \mathrm{d}_{\mathrm{i}}=0$ ). In both lines the difference between the experimental data and prediction increases with axial distance. This under prediction could be due to the difference of the turbulent intensity, between the experiments and simulation, at the jet inlets. The under prediction of turbulent quantities at the jet exit could be either due to the lack of turbulence level information from the experiments or due to the turbulent closure modeling. From all the above results it is evident that the RANS simulations are able to predict the trend and the magnitude at a reasonable level. Having these in mind, the numerical simulations are extended further to study the influence of discrete particles on the continuous phase. This is discussed next.
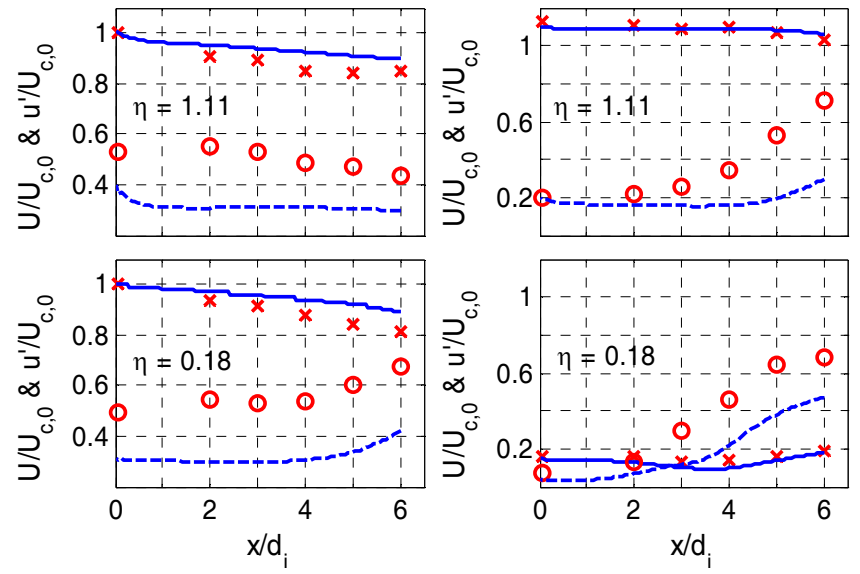

Figure 7: Comparison of axial velocity and axial turbulent intensity decay along the centerline of the inner (left) and outer (right) jets. ( $\mathrm{x}$ ) $\operatorname{Expt}\left(\mathrm{U} / \mathrm{U}_{\mathrm{c}, 0}\right) ;(\mathrm{o}) \operatorname{Expt}\left(6^{*} \mathrm{u}^{\prime} / \mathrm{U}_{\mathrm{c}, 0}\right) ;$ continuous line - Num $\left(\mathrm{U} / \mathrm{U}_{\mathrm{c}, 0}\right)$ and dashed line - Num $\left(6^{*} \mathrm{u}^{\prime} / \mathrm{U}_{\mathrm{c}, 0}\right)$. Here, $\mathrm{U}_{\mathrm{c}, 0}$ are the inner jet velocity at $\mathrm{x} / \mathrm{d}_{\mathrm{i}}=0$.

\subsection{Two phase flow}

In this section results pertaining to the two phase simulations are presented and discussed. The effect of discrete particles on the continuous phase flow field is 
discussed by comparing the two phase results with the single phase results. Although the simulations are carried out at two mass loading ratios, the difference in the predictions between the mass loadings are marginal and hence not presented here for the sake of brevity. The high mass loading results are compared with the single phase results.

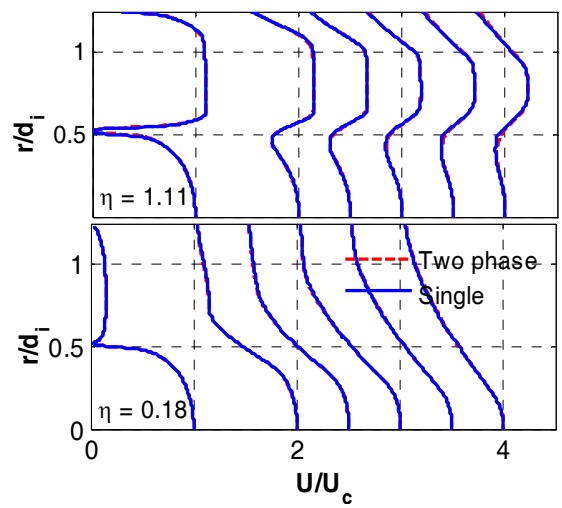

Figure 8: Comparison of the radial variation of axial velocity predictions of two phase and single phase at different axial locations (from left $\mathrm{x} / \mathrm{d}_{\mathrm{i}}=0.07,2,3,4,5$ and 6 ).

Figure 8 shows the variation of the axial velocity component along the radial direction predicted with and without the discrete particles. The rate of decrease in continuous-phase centerline velocity is seen to be similar in the single phase as well as in two phase flow predictions. It is evident from fig. 8 that overall the difference between the dispersed phase and the single phase predictions is almost nil except a few regions near the inner shear layer at $\eta=1.11$. This is found to be consistent with the experimental observation by Arai et al. [23], and later, Sadr and Klewicki [17], and Virdung and Rasmuson [8] wherein the effect of mass loading at $0.21 \%$ on the continuous phase was reported as insignificant.

Figure 9 shows comparison of the axial turbulent intensity predicted along the radial direction with and without the dispersed phase. It is observed that at $\eta=$ 1.11 , the turbulent intensity gradually decreases in the outer mixing region with increase in axial distance, whereas, the axial turbulent intensity is considerably affected in the inner jet alone as the particles are injected in the inner jet. At $\mathrm{x} / \mathrm{d}_{\mathrm{i}}$ $=0.07$, the axial turbulent intensities predicted (in the inner jet region) with the dispersed phase are lower than the single phase predictions at all velocity ratios. This can be attributed to the fact that the particles seem to attenuate the continuous phase turbulent intensities as experimentally observed by Fan et al. [24]. Further downstream, the dispersed phase turbulent intensity is marginally lower than single phase only in the outer mixing region elsewhere; the values are comparable to the single phase results. Overall, the difference in turbulent intensity between the dispersed phase and the single phase is predicted to be high 
only in the immediate vicinity of the jet exit. Far downstream, the difference is marginal. Gore and Crowe [25] related such a trend for the small particle size, and low stokes number, which is the case in this study.
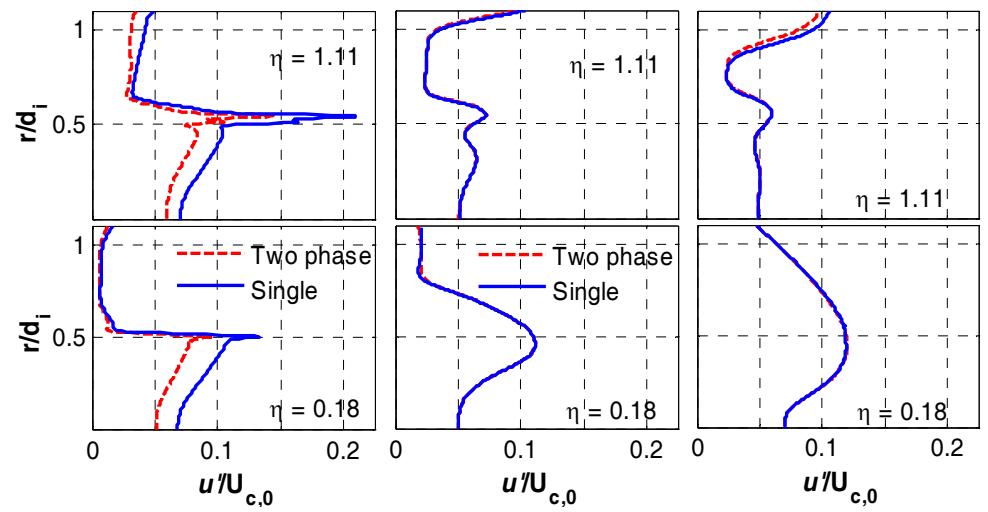

Figure 9: Comparison of axial intensity at stream-wise locations $\mathrm{x} / \mathrm{d}_{\mathrm{i}}=0.07$ (left), 3 (center) and 6 (right) at different velocity ratios.

Figure 10 shows the comparison of the predictions of correlation coefficient, $C_{u v}=\overline{u v} /\left(u^{\prime} v^{\prime}\right)$ with and without the dispersed phase. The predictions are different only in a few regions in the inner mixing region elsewhere, the difference is insignificant. This corroborates with the fact that the axial and radial turbulent intensities are almost the same and marginally different from the single phase predictions. At $\eta=0.18$, the difference is almost nil at all axial locations investigated here.
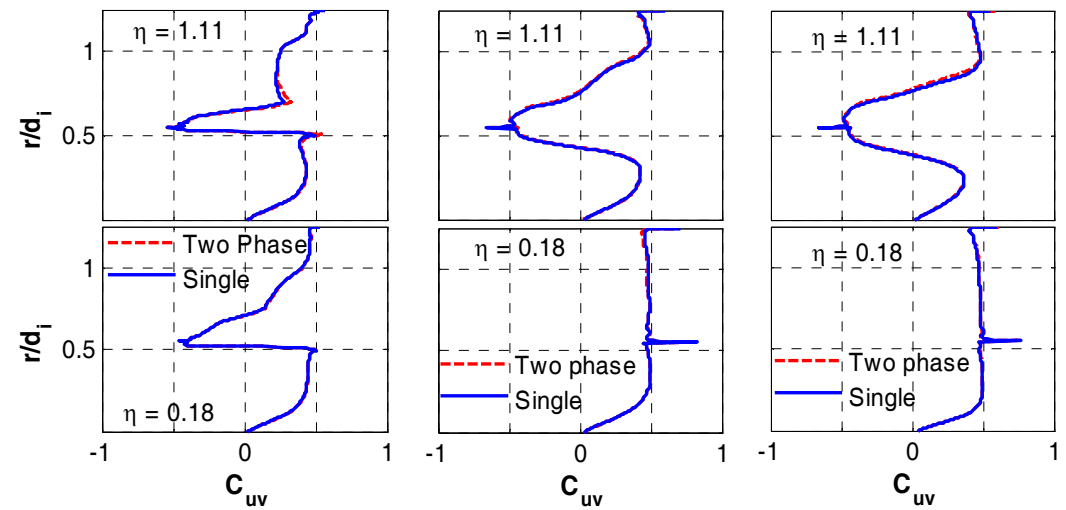

Figure 10: Comparison of correlation coefficient at stream-wise locations $\mathrm{x} / \mathrm{d}_{\mathrm{i}}$ $=0.07$ (left), 3.67 (center) and 6 (right) at different velocity ratios for the single and two phase flows. 
In addition to the axial velocity and turbulent intensity, the comparison of the vorticity field between the single-phase and two-phase results is also discussed. Since the difference between two phase and single phase predictions is small, only the normalized difference is presented instead of the absolute values to facilitate the discussion. The vorticity is normalized using the central jet velocity and central jet diameter. Then the difference, $\Delta \xi_{21}$ is calculated between the single phase and two phase conditions. The difference, $\Delta \xi_{21}$ at a given condition is then normalized using the maximum value of the difference, $\left(\Delta \xi_{21}\right)_{\max }=6.1$ at $\eta=1.11$. Hence, the difference presented here is the difference between the normalized vorticities not the difference in absolute value of vorticity.

Figure 11 shows the normalized vorticity difference between two-phase and single-phase. It is worth noting that at $\mathrm{x} / \mathrm{d}_{\mathrm{i}}=0.07$ in the inner mixing region, the maximum difference is in the negative direction implying that the single phase prediction is higher than the two phase prediction at $\eta=1.11$. This in turn implies that the particles in the continuous phase influence adversely in the inner side of the inner mixing region. However, the peak in the outer side of the inner mixing region is positive when compared to the inner side. This could be due to the restricted spread of the inner jet and in turn the particles by the outer jet.

At low velocity ratio $\eta=0.18$, the difference is only marginal which indicates that the fluid rotation is not affected by the presence of particles. However, at downstream locations, the difference increases gradually. This could be due to the effect of the solid particles on the entrainment in the outer jet at $\eta=0.18$. At $\mathrm{x} / \mathrm{d}_{\mathrm{i}}=0.07$, the difference is seen to increase with increase in velocity ratio. At $\mathrm{x} / \mathrm{d}_{\mathrm{i}}=6$, the difference is high at low velocity ratio when compared to $\eta=1.11$. This is due to the entrainment of the outer jet into the inner jet. Interestingly, from this trend it can be inferred that the particles in the inner mixing region tend to reduce the fluid rotation possibly owing to the transfer of momentum from the continuous phase to the particles. The influence of particles on the fluid rotation is minimal at all conditions. Away from the jet exits, the difference is seen to decrease at both velocity ratios.
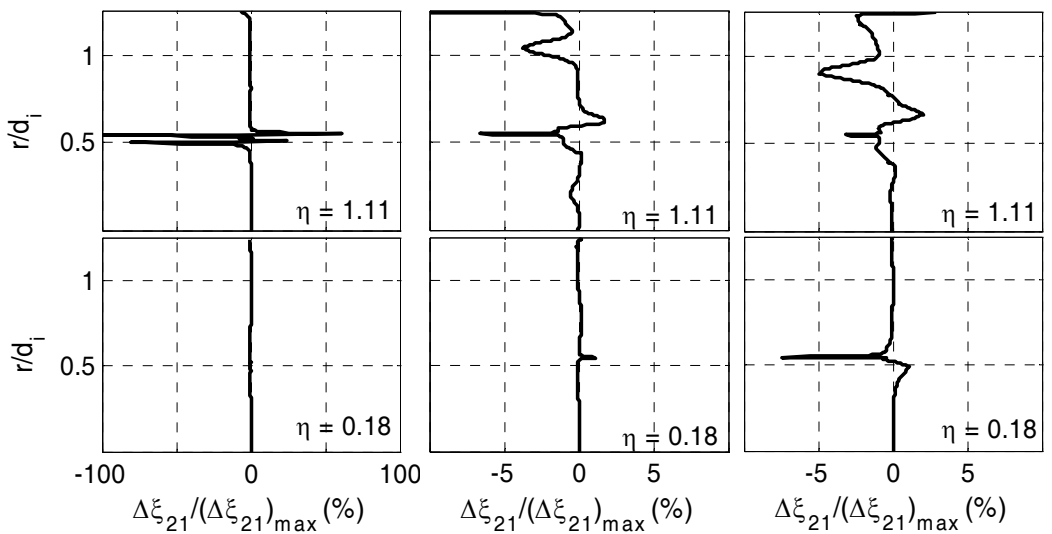

Figure 11: Variation of vorticity difference at different axial locations, $\mathrm{x} / \mathrm{d}_{\mathrm{i}}=$ 0.07 (left), 3 (center) and 6 (right). 
All these results clearly demonstrate that velocity ratio has a major contribution in the development of coaxial jet flow field. Furthermore, the addition of discrete particles alters the continuous phase flow field. However, it is evident only in the turbulent quantities rather than the mean quantities.

\section{Conclusions}

In this work, the flow field dynamics of the particle laden coaxial, turbulent jets are numerically investigated at two different velocity ratios. To this end, 3D, incompressible discrete phase turbulent flow calculations are carried out. In this study, glass beads of $240 \mu \mathrm{m}$ diameter are injected in the inner jet alone. For the mass loading studied in this work, the overall difference in the prediction between two phase and single phase is seen to be minimal which in turn implies that the influence of discrete particles on the continuous phase flow field is insignificant. However, close to the jet exits, the presence of discrete particles is seen to attenuate the continuous phase turbulence along the pipe. The results from this study demonstrate that the velocity ratio is an important parameter in the development of coaxial jet flow field and particles (of the size considered) will have an influence on the continuous phase only in the near flow field of the coaxial jets.

\section{Acknowledgement}

This work was partially supported by Qatar Science and Technology Park.

\section{References}

[1] Ko, N.W.M. and Kwan, A.S.H., The initial region of subsonic coaxial turbulent jets, Journal of Fluid Mechanics, 73, pp. 305-332, 1976.

[2] Dahm, W.J.A., Frieler, C.E. and Tryggvason, G., Vortex structure and dynamics in the near field of a coaxial jet, Journal of Fluid Mechanics, 241, pp. 371-402, 1992.

[3] Buresti, G., Petagna, P. and Talamelli, A., Experimental investigation on the turbulent near-field of coaxial jets, Experimental Thermal and Fluid Sciences, 17, pp. 18-36, 1998.

[4] Balarac, A., Si-Ameur, M., Lesieur, M. and Metais, O., Direct numerical simulations of high velocity ratio coaxial jets: mixing properties and influence of upstream conditions, Journal of Turbulence, 8, pp. 1-14, 2007.

[5] Mostafa, A.A., Mongia, H.C., McDonell, V.G. and Samuelsen, G.S., An experimental and numerical study of particle-laden coaxial jet flows, International Journal of Heat and Fluid Flow, 11 (2), pp. 90-97,1990.

[6] Fan, J., Zhao, H. and Jin, J., Two-phase velocity measurement in particleladen coaxial jets, Chemical Engineering Journal, 63, pp. 11-17, 1996. 
[7] Fan, J., Zhang, X., Chen, L. and Cen, K., New stochastic particle dispersion modeling of a turbulent particle-laden round jet, Chemical Engineering Journal, 66, pp. 207-215, 1997.

[8] Virdung, T. and Rasmuson, A., Hydrodynamic properties of a turbulent confined solid-liquid jet evaluated using PIV and CFD, Chemical Engineering Science, 62, pp. 5963-5978, 2007.

[9] Brucato, A., Grisafi, F. and Montante, G., Particle drag coefficients in turbulent fluids, Chemical Engineering Science, 53, pp. 3295-3314, 1998.

[10] Stakic, M.B., Zivkovic, G.S. and Sijercic, M.A., Numerical analysis of discrete phase influence on gas flow in a two-phase turbulent free jet, Proc. of the ASME FED, FED-244, pp. 501-506, 1997.

[11] Sijercic, M., Belosevic, S. and Stevanovic, Z., Simulation of free turbulent particle-laden jet using Reynolds-stress gas turbulence model, Applied Mathematical modeling, 31, pp. 1001-1014, 2007.

[12] Liu, Y., Zhou, L.X. and Xu, C.X., Numerical simulation of instantaneous flow structure of swirling and non-swirling coaxial jet particle-laden turbulence flows, Physica A, 389, pp. 5380-5389, 2010.

[13] Ahmed, A.M. and Elghobashi, S., Direct numerical simulation of particle dispersion in homogeneous turbulent shear flows, Physics of Fluids, 13, pp. 3346-3364, 2001.

[14] Sadr, R. and Klewicki, J.C., An experimental investigation of the near-field flow development in coaxial jets, Physics of Fluids, 15, pp. 1233-1246, 2003.

[15] Sadr, R. and Klewicki, J.C., A Multi Purpose Feed System for Fluids and Solid Particles, Measurement Science and Technology, 14, N33-N35, 2003.

[16] Koochesfahani, M.M. and Nocera, D.G., Handbook of Experimental Fluid Dynamics, Springer-Verlag, Chapter 5.4, 2007.

[17] Sadr, R. and Klewicki, J.C., Flow field characteristics in the near field region of particle-laden coaxial jets, Experiments in Fluids, 39, pp. 885894, 2005.

[18] Menter, F.R., Zonal Two Equation $k$ - $\omega$ Models for Aerodynamic Flows, AIAA Paper 93-2906, 1993.

[19] Shih, T.H., Liou, W.W., Shabbir, A., Yang, Z. and Zhu, J., A New k- $\varepsilon$ Eddy-Viscosity Model for High Reynolds Number Turbulent Flows Model Development and Validation, Computers Fluids, 24 (3), pp. $227-$ 238, 1995.

[20] Hardalupas, Y., Taylor, A.M.K.P. and Whitelaw, J.H., Velocity and particle flux characteristics of turbulent particle-laden jets, Proc. of Royal Society London A, 426, pp. 31-78, 1989.

[21] Roe, P.L., Characteristic based schemes for the Euler equations, Annual Review of Fluid Mechanics, 18, pp. 337-365, 1986.

[22] ANSYS - Fluent 12.0, 2010.

[23] Arai, T., Kudo, N., Ishima, T., Youssef, I.M. and Obokata, T., Turbulence structure of a liquid-solid two-phase jet by means of laser techniques. In: Proc. of $4^{\text {th }}$ ASME-JSME Conference, USA, July 6-10,pp. 847-853, 2003. 
[24] Fan, J., Zhao, H. and Cen, K., An experimental study of two-phase turbulent coaxial jets, Experiments in Fluids, 13, pp. 279-287, 1992.

[25] Gore, R.A. and Crowe, C.T., Effect of particle size on modulating turbulent intensity. International Journal of Multiphase Flow, 15 (2), pp. 279-285, 1989. 